\title{
PHASE TRANSITIONS OF INTERACTING PARTICLE SYSTEMS
}


This page is intentionally left blank 


\title{
PHASE TRANSITIONS OF INTERACTING PARTICLE SYSTEMS
}

\section{Norio Konno}

\author{
Muroran Institute of Technology, Japan
}




\section{Published by}

World Scientific Publishing Co. Pte. Ltd.

P O Box 128, Farrer Road, Singapore 9128

USA office: Suite 1B, 1060 Main Street, River Edge, NJ 07661

UK office: 73 Lynton Mead, Totteridge, London N20 8DH

\section{PHASE TRANSITIONS OF INTERACTING PARTICLE SYSTEMS}

Copyright () 1994 by World Scientific Publishing Co. Pte. Ltd.

All rights reserved. This book, or parts thereof, may not be reproduced in any form or by any means, electronic or mechanical, including photocopying, recording or any information storage and retrieval system now known or to be invented, without written permission from the Publisher.

For photocopying of material in this volume, please pay a copying fee through the Copyright Clearance Center, Inc., 27 Congress Street, Salem, MA 01970, USA.

ISBN 981-02-2076-6 


\section{To my parents:}

Kazue and Masako 
This page is intentionally left blank 


\section{PREFACE}

Recently interacting particle systems have been studied widely from many aspects, for examples, mathematics, physics, chemistry, and biology. Many researchers get interested in this field. Fortunately some good books on it have been published, for example, Liggett (1985) Interacting Particle Systems, Durrett (1988) Lecture Notes on Particle Systems and Percolation.

This book restricts attention to the phase transitions of the interacting particle systems, in particular, their critical values and order parameters. As for concrete systems, we study basic contact processes, 0 -contact processes, diffusive contact processes, long range contact processes, and uniform nearest-particle systems. Unfortunately, with the exception of the critical value of the uniform nearest-particle system with infinite range, the rigorous critical values and order parameters are not known. So we turn our attention to the bounds on the critical values and the order parameters. Then our basic question in this book is as follows :

How can we get good bounds on the critical values and the order parameters?

The above question is very basic and many researchers have been trying to get better bounds rigorously. So we give not only our bounds but also other's ones in this book.

In the introductory Chapter 1 we consider briefly Markov chains and give some examples. 
The basic contact process is a continuous-time Markov process on state space $\{0,1\}^{\mathbf{Z}^{d}}$, where $\mathbf{Z}^{d}$ is the $d$-dimensional integer lattice. This process is interpreted as a simple model of a disease with the infection rate $\lambda$. The value 0 is regarded as a healthy individual while the value 1 is regarded as an infected one. The dynamics are specified by the following transition rates : for $\eta \in\{0,1\}^{\mathbf{Z}^{d}}$, at site $x \in \mathbf{Z}^{d}$,

$$
\begin{aligned}
& 1 \rightarrow 0 \quad \text { at rate } 1, \\
& 0 \rightarrow 1 \quad \text { at rate } \quad \lambda \times \sum_{y:|y-x|=1} \eta(y) \text {, }
\end{aligned}
$$

where $|x|=\left|x_{1}\right|+\cdots+\left|x_{d}\right|$. Define $\rho_{\lambda}$ as the density of infected individual at a site with respect to a stationary measure starting from $\eta \equiv 1$. We take $\rho_{\lambda}$ as an order parameter of the basic contact process. The basic contact process is attractive, so $\rho_{\lambda}$ is a nondecreasing function of $\lambda$. Then the critical value $\lambda_{c}$ is characterized by $\rho_{\lambda}$ in the following way :

$$
\begin{aligned}
\lambda_{c} & =\sup \left\{\lambda \geq 0: \rho_{\lambda}=0\right\} \\
& =\inf \left\{\lambda \geq 0: \rho_{\lambda}>0\right\} .
\end{aligned}
$$

As for the processes treated in the rest of this book, the critical values and the order parameters can be defined in the similar way, since these processes are also attractive. Then $\lambda_{c}$ and $\rho_{\lambda}$ are not known rigorously. Chapter 2 contains the one-dimensional basic contact process on finite sets. Chapter 3 is devoted to lower bounds on the critical value and upper bounds on the order parameter of basic contact process. In Section 3.3 using the correlation identities and the Harris-FKG inequality we give the lower bounds on the critical value and upper bounds on the order parameter. Better bounds are obtained by the Harris lemma in Section 3.4. Section 3.5 contains the best lower bound on the critical value of the one-dimensional basic contact process given by Liggett and Ziezold-Grillenberger methods. In particular, we clarify the relation between both methods. On the other hand Chapter 4 treats the upper bounds on the critical value and lower bounds on the order parameter of the one-dimensional basic contact process by various methods.

The $\theta$-contact process is a generalization of the one-dimensional basic 
contact process. The evolution mechanism is as follows :

\begin{tabular}{|c|c|}
\hline $1 \rightarrow 0$ & at rate \\
\hline $001 \rightarrow 011$ & at rate \\
\hline $100 \rightarrow 110$ & at rate \\
\hline $101 \rightarrow 111$ & at rate \\
\hline
\end{tabular}

where $\theta \geq 0$. Remark that the process for $\theta=2$ is nothing but the basic contact process in one dimension. The first three sections in Chapter 5 we restrict attention to a family of the $\theta$-contact processes for $1 \leq \theta \leq 2$. This restriction comes from the existence of a coalescing duality for this process. After that we will discuss general case, $\theta \geq 1$, by using the Gray duality. In Section 5.2 we give lower bounds on the critical value $\lambda_{c}(\theta)$ for $1 \leq \theta \leq 2$ by using the Griffeath method and the Liggett and Ziezold-Grillenberger methods. Section 5.3 is devoted to upper bounds derived from the HolleyLiggett method for $1 \leq \theta \leq 2$. In Section 5.4 we introduce the coalesing duality and discuss about some examples. The Gray duality is presented in Section 5.5. So using it, we will give lower bounds on $\lambda_{c}(\theta)$ for $\theta \geq 1$.

The diffusive contact process is the basic contact process with stirring mechanism which is given as follows : for each $x, y \in \mathbf{Z}^{d}$ with $|x-y|=1$, we exchange the values at $x$ and $y$ at rate $D$. Then as in the case of the basic contact process the order parameter $\rho_{\lambda}(D)$ and critical value $\lambda_{c}(D)$ can be defined. In this setting it is known that $\rho_{\lambda}(D) \rightarrow(2 d \lambda-1) / 2 d \lambda$ and $\lambda_{c}(D) \rightarrow 1 / 2 d$ as $D \rightarrow \infty$. In Chapters 6 and 7 we mainly prove that there are positive constants $C_{1}, C_{2}$ so that $C_{1} \varphi_{d}(D) \leq \lambda_{c}(D)-1 / 2 d \leq$ $C_{2} \varphi_{d}(D)$ when $D$ is large, where $\varphi_{1}(D)=1 / D^{1 / 3}, \varphi_{2}(D)=|\log D| / D$, and $\varphi_{d}(D)=1 / D(d \geq 3)$. Chapter 6 is devoted to proofs of the upper bounds on $\lambda_{c}(D)-1 / 2 d$. Moreover we give another proof of $\rho_{\lambda}(D) \rightarrow(2 d \lambda-1) / 2 d \lambda$ and show crossover phenomena on $\rho_{\lambda}(D)$. On the other hand Chapter 7 treats the proofs of the lower bounds on $\lambda_{c}(D)-1 / 2 d$.

As in the diffusive contact process, we study the long range contact process in Chapter 8.

The uniform nearest-particle system with the infection rate $\lambda$ and the distance of range $M$ is the one-dimensional interacting particle system. In particular, if $M=1$, then this system is equivalent to the basic contact process in one dimension. In Chapter 9 we consider uniform nearset-particle systems with finite and infinite ranges respectively. Section 9.2 contains the lower bounds on critical values and upper bounds on order parameters when 
$M$ is finite. In the case of infinite range, the rigorous critical value is known. So we give a good upper bound on order parameter near critical value in Section 9.3 .

Finally Chapter 10 gives a survey of results on diffusive contact processes and diffusive branching processes on the homogeneous tree.

I express special thanks to Tokuzo Shiga for introducing me to this fascinating field and constantly encouraging me throughout the whole work. I am deeply indebted to Makoto Katori, for teaching me much about statistical physics while collaborating on various projects. Moreover Makoto Katori read various parts of the manuscript and made numerous suggestions for improvements. I extend our thanks to all these, and to others : Rick Durrett, Tom Liggett, and Roberto Schonmann, who have offered advice in correspondence or conversation. But I am, of course, responsible for all errors that remain.

Most of this book was written while I was visiting the Mathematical Sciences Institute at Cornell University during May 1993 - April 1994, a visit assisted by a grant from the Ministry of Education of Japan. I am grateful for not only this grant but also the financial support of the Army Research Office (through the Mathematical Sciences Institute at Cornell University). Moreover it is a pleasure to acknowledge the passive and active assistance of Rick Durrett during this period.

Finally I would like to thank my wife, Naomi, and my two daughters, Yuki and Aki, for their patience and understanding.

N.K.

Muroran August 1994 


\section{CONTENTS}

Preface . . . . . . . . . . . . . . . . . vii

\section{CHAPTER 1. INTRODUCTION - MARKOV CHAINS}

1.1. Introduction . . . . . . . . . . . . . . . . . . . . . . 1

1.2. Discrete-Time Markov Chains . . . . . . . . . . . . . . 1

1.3. Continuous-Time Markov Chains . . . . . . . . . . . . 5

CHAPTER 2. CONTACT PROCESS TYPE MODELS ON SMALL FINITE SETS

2.1. Introduction . . . . . . . . . . . . . . . . . . . . . . 7

2.2. The Basic Contact Process on Finite Set . . . . . . . . . 7

2.2.1. $n=1$. . . . . . . . . . . . . . . . . . . . . . 7

2.2.2. $n=2$. . . . . . . . . . . . . . . . . . . . . . . 8

2.2.3. $n=3$. . . . . . . . . . . . . . . . . . . . . . . 9

2.2.4. General case . . . . . . . . . . . . . . . . . . . . 9 
2.3. The Threshold Contact Process on Finite Set

\section{CHAPTER 3. THE BASIC CONTACT PROCESS I}

3.1. Introduction . . . . . . . . . . . . . . . . . . . . . 15

3.2. Correlation Identities . . . . . . . . . . . . . . . . . 18

3.3. Harris-FKG Inequality Method . . . . . . . . . . . . 21

3.3.1. First bound . . . . . . . . . . . . . . . . . . . . 23

3.3.2. Second bound . . . . . . . . . . . . . . . . . 24

3.3.3. Third bound . . . . . . . . . . . . . . . . . . 24

3.3.4. Fourth bound . . . . . . . . . . . . . . . . 25

3.3.5. Higher dimensions . . . . . . . . . . . . . . . . 26

3.3.6. Summary . . . . . . . . . . . . . . . . . . . . . 27

3.4. Harris Lemma and Markov Extension Method . . . . 27

3.4.1. Harris lemma . . . . . . . . . . . . . . . . . . . 28

3.4.2. First bound by the Harris lemma $\quad$. . . . . . . . . . . . 29

3.4.3. Second bound by the Harris lemma . . . . . . . . 30

3.4.4. Third bound by the Harris lemma . . . . . . . . . . 30

3.4.5. Higher dimensions . . . . . . . . . . . . . . . . . 31

3.4.6. Remark . . . . . . . . . . . . . . . . . . . . . 32

3.5. Liggett and Ziezold-Grillenberger Methods . . . . . . 33

3.5.1. $n=1$. . . . . . . . . . . . . . . . . . . . 36

3.5.2. $n=2 \quad$. . . . . . . . . . . . . . . . . . . . 37

3.5.3. General case . . . . . . . . . . . . . . . . . . 38

3.6. Application to the Threshold Contact Process . . . . . 41

3.6.1. Introduction . . . . . . . . . . . . . . . . . . . 41

3.6.2. Liggett and Ziezold-Grillenberger methods revisited . . 42

3.6.3. General case . . . . . . . . . . . . . . . . . . 45

3.6.4. Lower bounds . . . . . . . . . . . . . . . . . . . 48

3.7. Summary . . . . . . . . . . . . . . . . . . . . 50

CHAPTER 4. THE BASIC CONTACT PROCESS II 
4.2. Contour Method . . . . . . . . . . . . . . . . . . . 53

4.2.1. Oriented bond percolation . . . . . . . . . . . . . 53

4.2.2. Oriented site percolation . . . . . . . . . . . . . . 56

4.3. Comparison Method . . . . . . . . . . . . . . . . . 57

4.4. Holley-Liggett Method . . . . . . . . . . . . . . . . 59

4.5. Correlation Identities I . . . . . . . . . . . . . . . . 59

4.6. Correlation Identities II . . . . . . . . . . . . . . . . 62

4.7. Decoupling Procedure : Nonrigorous Results . . . . . 66

4.7.1. Renewal decoupling . . . . . . . . . . . . . 66

4.7.2. A new decoupling . . . . . . . . . . . . . . . . . 67

4.8. Remark . . . . . . . . . . . . . . . . . . . . . . 69

CHAPTER 5. THE $\theta$-CONTACT PROCESS

5.1. Introduction . . . . . . . . . . . . . . . . . . 70

5.2. Lower Bounds . . . . . . . . . . . . . . . . . . . . . 75

5.2.1. Griffeath method . . . . . . . . . . . . . . . . . 75

5.2.2. Liggett and Ziezold-Grillenberger methods . . . . . . 78

5.3. Upper Bounds . . . . . . . . . . . . . . . . . . . . 80

5.4. Coalescing Duality . . . . . . . . . . . . . . . . . . 84

5.5. Gray Duality . . . . . . . . . . . . . . . . . . . . . 93

\section{CHAPTER 6. DIFFUSIVE CONTACT PROCESSES I}

6.1. Introduction . . . . . . . . . . . . . . . . . . . . . 100

6.2. Diffusive Contact Processes on Small Finite Sets . . . 104

6.2.1. $n=1$.. . . . . . . . . . . . . . . . . . . 104

6.2.2. $n=2$. . . . . . . . . . . . . . . . . . . 105

6.2.3. $n=3$. . . . . . . . . . . . . . . . . . 105

6.2.4. Summary . . . . . . . . . . . . . . . . . . . 106

6.2.5. General case . . . . . . . . . . . . . . . 107

6.3. Preliminary Results . . . . . . . . . . . . . . . . . 109

6.4. Upper Bounds . . . . . . . . . . . . . . . . . . . . . 119

6.5. Proofs of Theorems 6.1.1 (2) and 6.1.3 . . . . . . . . . 132 
6.5.1. Proof of Theorem $6.1 .1(2)$. . . . . . . . . . . . . . . 132

6.5.2. The Galton-Watson process . . . . . . . . . . . . . 138

6.5.3. Proof of Theorem 6.1.3 . . . . . . . . . . . . . . . . . . 140

CHAPTER 7. DIFFUSIVE CONTACT PROCESSES II

7.1. Introduction . . . . . . . . . . . . . . . . . . . . . 146

7.2. Lower Bounds for $d=1$. . . . . . . . . . . . . . . . 146

7.3. Lower Bounds for $d=2$. . . . . . . . . . . . . . . . 151

7.4. Lower Bounds for $d \geq 3$. . . . . . . . . . . . . . . 166

\section{CHAPTER 8. LONG RANGE CONTACT PROCESSES}

8.1. Introduction . . . . . . . . . . . . . . . . . . . . . 171

8.2. Preliminary Results . . . . . . . . . . . . . . . . . . 175

8.3. Upper Bounds . . . . . . . . . . . . . . . . . . . . . . 182

8.4. Lower Bounds for $d=1$. . . . . . . . . . . . . . . . 192

8.5. Lower Bounds for $d=2$. . . . . . . . . . . . . . . . 194

8.6. Lower Bounds for $d \geq 3$. . . . . . . . . . . . . . . . 197

\section{CHAPTER 9. UNIFORM NEAREST-PARTICLE SYSTEMS}

9.1. Introduction . . . . . . . . . . . . . . . . . . . . . 199

9.2. Uniform Nearest-Particle Systems : Finite Ranges . . 199

9.2.1. Attractive nearest-particle systems with finite ranges . . 200

9.2.2. Better lower bounds . . . . . . . . . . . . . . . 207

9.2.3. UNPS $(M)$. . . . . . . . . . . . . . . . . . . . 209

9.3. Uniform Nearest-Particle Systems : Infinite Ranges . . 210 
9.4. UNPS $(M)$ (Finite $M$ and Finite System) . . . . . . . 212

CHAPTER 10. PARTICLE SYSTEMS ON TREES

10.1. Introduction . . . . . . . . . . . . . . . . . . . 214

10.2. Preliminary Results . . . . . . . . . . . . . . . . . 219

10.3. $\vec{\lambda}_{c}(D)$. . . . . . . . . . . . . . . . . . . . 223

10.4. Lower Bounds on $\lambda_{c}^{g}(D)$. . . . . . . . . . . . . . . 224

Subject Index . . . . . . . . . . . . . . . . . . . . . . . 226 